\title{
Characterization of LhSorTGA2, a novel TGA2-like protein that interacts with LhSorNPR1 in oriental hybrid lily Sorbonne
}

\author{
Le Wang ${ }^{1,2}$, Zhihong Guo ${ }^{1}$, Yubao Zhang ${ }^{1}$, Yajun Wang ${ }^{1}$, Li Wang ${ }^{3}$, Guo Yang ${ }^{1}$, Wenmei Li ${ }^{1,2}$, Ruoyu Wang ${ }^{1}$ \\ and Zhongkui Xie ${ }^{1 *}$
}

\begin{abstract}
Background: Non-expressor of pathogenesis-related genes 1 (NPR1) regulates expression of pathogenesis-related $(P R)$ genes by interacting with TGA family proteins during systemic acquired resistance (SAR). However, no TGA-like proteins or their interacting partners have been characterized in lily.

Results: In the present study, LhSorTGA2, a novel TGA-like protein, was identified as an interacting partner of LhSorNPR1 (an NPR-like protein) by bimolecular fluorescence complementation (BIFC) and yeast two-hybrid assay (Y2H). Subcellular localization of GFP-tagged proteins targeted LhSorTGA2 to the nucleus, whereas GFP-labeled LhSorNPR1 was observed both in the nucleus and at the cytomembrane. Sequence alignment revealed that LhSorTGA2 was featured with a basic leucine zipper (bZIP) domain and two glutamine rich acid domains (QI and QII). Further phylogenetic analysis showed that TGA family proteins can be grouped into three subclades, within which LhSorTGA2 was clustered into subclade I, together with AtTGA2/5/6. Expression of LhSorTGA2 was investigated in different tissues by qPCR, and the highest expression level was observed in stem. Besides, when treated with phytohormones (SA, MeJA, ETH and ABA) or fungal pathogen Botrytis elliptica, LhSorTGA2 expression was also induced at different time points post treatments.
\end{abstract}

Conclusions: Collectively, these results suggested that LhSorTGA2 was an interacting partner of LhSorNPR1, which might function in regulating expression of $P R$ genes in lily during SAR.

Keywords: Non-expressor of pathogenesis-related genes 1,TGA-like proteins, Protein interaction, Gene expression, Subcellular localization, Lilium spp.

\section{Background}

Infections at local sites often induce resistance against further invasions of pathogenic organisms in the distal uninfected parts of plants. This primed resistance, which protects plants from further invasion of a broad spectrum of pathogens at the whole-plant level, is referred to as systemic acquired resistance (SAR) (Fu and Dong 2013). The establishment of SAR is accompanied by accumulation of salicylic acid (SA) (Gao et al. 2015), and induction

\footnotetext{
*Correspondence: wxhcas@|zb.ac.cn

${ }^{1}$ Gaolan Station of the Agricultural and Ecological Experiment, Northwest Institute of Eco-Environment and Resources, Chinese Academy of Sciences, Lanzhou 730000, China

Full list of author information is available at the end of the article
}

of pathogenesis-related (PR) genes. PRs encode for small proteins, of which some are endowed with direct antimicrobial activities. Since SA level, PR accumulation, and SAR are tightly linked, SAR-conferred resistance could be severely compromised either by interfering the level of SA synthesis or by disrupting signaling pathways control $P R$ gene expression (Gaffney et al. 1993). Mutation of the non-expressor of PR genes 1 (NPR1) gene, a central node in SA-mediated defense signaling, blocks the priming of SAR (Cao et al. 1997). NPR1 lacks a DNA binding domain itself, but features with an ankyrin repeat domain and a BTB/POZ domain, both of which mediate protein-protein interaction (Pieterse and Van Loon 2004). The ankyrin repeat at C-terminus interacts with TGA2 
to activate $P R 1$ transcription, which is critical to plant defense against biotrophic pathogens in the state of SAR (Fan and Dong 2002; Zhang et al. 1999).

TGA proteins are members of the group $D$ basic region/leucine zipper (bZIP) transcription factors (TFs) (Jakoby et al. 2002), which recognize the TGACG motif (also known as activation sequence 1; as-1) in the promoter region (Gatz 2013). With the identification of as-1 elements in promoters of a number of genes, the crucial roles TGA TFs played in gene expression regulation were unveiled. Mutagenesis of LS7 element, which contained a TGA-binding site, resulted in complete abolishment of PR1 expression. On the contrary, mutation of LS5, another TGACG motif containing element, augmented PR1 expression (Kesarwani et al. 2007). The opposite effects exerted by these two TGACG motifs to PR1 expression implied that transcription of genes containing as-1 elements in the adjacent promoter, might subject to complex regulation by different TGAs in the genome.

Lilies are herbaceous perennials that renowned for their elegant flowers. However, bulb and cut flower production in genus Lilium, are both under severe threat of pathogens in different types. For instance, lily bulbs can be infected by some soil-borne fungal pathogens (Fusarium oxyporum, Penicillium albocoremium, and Penicillium tulipae), causing rot of scales, bulb bases, and buds (Lecomte et al. 2016; Wang et al. 2017a). Furthermore, infection of air-borne fungi, such as Botrytis cinerea and Botrytis elliptica, could lead to fire blight disease, which is destructive to cut flower production (Huang et al. 2012; Wang et al. 2017d). In addition, multiplication of viruses (lily symptomless virus, cucumber mosaic virus, and lily mottle virus) also affects plant growth, resulting in leaf mottle and contortion, as well as stunted growth of lily plants (Zhang et al. 2016). To counteract pathogenic organisms, a comprehensive understanding of defense related genes that govern plant immunity in lily would be of great necessity.

In the present study, LhSorTGA2, a TGA2-like protein that interacted with LhSorNPR1 (Wang et al. 2017b) was characterized. Subcellular localization revealed that presence of GFP-tagged LhSorNPR1 was detected both in the nucleus and at the cytomembrane, but the GFP-tagged LhSorTGA2 was predominantly detected in the nucleus. LhSorTGA2 expression in response to various phytohormone treatments and fungal pathogen B. elliptica infection was also explored.

\section{Methods}

\section{Plant material and treatments}

Bulbs (10-12 cm in diameter) of oriental hybrid cultivar 'Sorbonne' were planted in plastic pots containing peat moss as a growing substrate. All pots were then transferred to the growth chamber of Gaolan Station of Agricultural and Ecological Experiment, to keep plants under controlled conditions ( $16 \mathrm{~h} \mathrm{light} / 8 \mathrm{~h}$ dark; $22{ }^{\circ} \mathrm{C}$ ). Nicotiana benthamiana plants were raised under the same conditions for observation of protein localization in planta.

\section{Full-length cloning of $L h S o r T G A 2$}

Total RNA was extracted from the leaves of 1-monthold seedlings using the RNAprep Pure Kit for plants (TIANGEN Corporation, Beijing, China). Partial coding sequence of LhSorTGA2, which was annotated in our previous RNA-seq data, was used to design gene specific primers (5RACE-1, 5RACE-2, 3RACE-1, 3RACE-2; Table 1) for rapid amplification of cDNA ends (RACE). $5^{\prime}$ RACE and $3^{\prime}$ RACE were conducted according to the instructions of the SMARTer ${ }^{\circledR}$ RACE $5^{\prime} / 3^{\prime}$ Kit (Takara, China). The cDNA full-length sequence of LhSorTGA2 was obtained by assembling the $5^{\prime}$-RACE sequence, the partial coding sequence, and the $3^{\prime}$-RACE sequence.

\section{In silico analysis of LhSorTGA2}

The open reading frame (ORF) of the assembled LhSorTGA2 full-length cDNA was predicted by ORF finder. The theoretical isoelectric point and molecular weight were calculated by using the Compute $\mathrm{pI} / \mathrm{Mw}$ tool of ExPASy. Sequences of TGA proteins were aligned with the Clustal W program. Construction of phylogenetic tree was carried out by Mega 5 software using the neighbor-joining method (Tamura et al. 2011).

\section{Subcellular localization and protein-protein interaction validated by BIFC}

The ORF of LhSorTGA2 without stop codon was amplified using primers LhSorTGA2-sub-F and LhSorTGA2sub-R, and inserted into SpeI digested pCAMBIA1302 vector to express LhSorTGA2 in frame with GFP at the $\mathrm{N}$-terminus. For the vector construction of LhSorNPR1, the ORF of LhSorNPR1 was amplified with primers LhSorNPR1-sub-F and LhSorNPR1-sub-R, and cloned into NcoI and SpeI digested PCAMBIA1302 vector after sequencing. The recombinant plasmids pCAMBIA1302LhSorTGA2 and pCAMBIA1302-LhSorNPR1 were each introduced into Agrobacterium tumefaciens (strain GV3101) competent cells via the freeze-thaw method. GV3101 cells harbouring pCAMBIA1302-LhSorTGA2 or pCAMBIA1302-LhSorNPR1 construct were infiltrated into leaf epidermal cells of 4-week-old $N$. benthamiana plants using a $1-\mathrm{ml}$ needleless syringe. The pCAMBIA1302 empty vector was used as the control for protein localization.

To validate the protein-protein interaction between LhSorTGA2 and LhSorNPR1, the bimolecular 
Table 1 List of primers used in the experiment

\begin{tabular}{|c|c|c|}
\hline Experiment & Primer name & Primer sequence $\left(5^{\prime}-3^{\prime}\right)$ \\
\hline \multirow[t]{2}{*}{ LhSorTGA2 5' RACE } & LhSorTGA2-5RACE-1 & CAGGTCAGCAGCATCCTCAATTCGG \\
\hline & LhSorTGA2-5RACE-2 & ATAGCCCCGAAGTCAGAAACCCGGG \\
\hline \multirow[t]{2}{*}{ LhSorTGA2 3' RACE } & LhSorTGA2-3RACE-1 & GAACCTCCAGCAGTCCTCCCAGCAG \\
\hline & LhSorTGA2-3RACE-2 & GGGTCAGATGGCTATGGCGATGGGA \\
\hline \multirow[t]{4}{*}{$\mathrm{BIFC}$} & LhSorTGA2-BIFC-F & CGTCTAGAATGCACTCCTCCCGGGTTTCT \\
\hline & LhSorTGA2-BIFC-R & CGGGTACCTTCCCGTGGACGGGCTAGCCA \\
\hline & LhSorNPR1-BIFC-F & CGGGGCCCATGGCCGACGCCGCCGAGTG \\
\hline & LhSorNPR1-BIFC-R & CGGGATCCTCATTCTTCCATATCTACCAGACA \\
\hline \multirow[t]{4}{*}{$\mathrm{Y} 2 \mathrm{H}$} & LhSorTGA2-GADT7-F & CGGAATTCATGCACTCCTCCCGGGTTTCT \\
\hline & LhSorTGA2-GADT7-R & CGGGATCCCTCATTCCCGTGGACGGGCTAG \\
\hline & LhSorNPR1-GBKT7-F & CGCCATGGAGATGGCCGACGCCGCCGA \\
\hline & LhSorNPR1-GBKT7-R & CGGGATCCCTCATTCTTCCATATCTACCAGACAG \\
\hline \multirow[t]{4}{*}{ Subcellular localization } & LhSorNPR1-sub-F & ATATCCATGGTAATGGCCGACGCCGCCGA \\
\hline & LhSorNPR1-sub-R & CGACTAGTTTCTTCCATATCTACCAGACAGACA \\
\hline & LhSorTGA2-sub-F & CGACTAGTATGCACTCCTCCCGGGTTTCTGA \\
\hline & LhSorTGA2-sub-R & CGACTAGTTTCCCGTGGACGGGCTAGCCA \\
\hline \multirow[t]{2}{*}{ LhSorTGA2 qPCR } & LhSor TGA2 qPCR-F & TCAGATGGCTATGGCGATGG \\
\hline & LhSor TGA2 qPCR-R & CGGATTGGCGAGTAGTGAGT \\
\hline
\end{tabular}

Restriction sites for vector construction are underlined

fluorescence complementation (BiFC) assay was performed using vectors described previously. LhSorNPR1 was constructed into the $\operatorname{PSPYNE}(\mathrm{R}) 173$ vector to express LhSorNPR1 in fusion with the N-terminus of YFP (LhSorNPR1-YFP $^{\mathrm{N}}$ ), and $L h S o r T G A 2$ was inserted into the pSPYCE(M) vector to express LhSorTGA2 in fusion with the C-terminus of YFP (LhSorTGA2-YFPC). The resulting recombinant plasmids, pSPYNE-LhSorNPR1 and pSPYCE-LhSorTGA2, were transformed into GV3101 and co-infiltrated into $N$. benthamiana leaf epidermal cells using a protocol described previously (Kerppola 2013). Fluorescence of YFP or GFP was observed $72 \mathrm{~h}$ post infiltration using a confocal laser scanning microscope (Leica TCS SP8, Leica Microsystems, Germany).

\section{Yeast two-hybrid assay}

To further confirm the protein interaction between LhSorTGA2 and LhSorNPR1, the ORF of LhSorNPR1 was amplified (LhSorNPR1-GBKT7F, LhSorNPR1-GBKT7-R; Table 1) and inserted into pGBKT7 vector to construct the pGBKT7-LhSorNPR1 bait plasmid. The prey plasmid, pGADT7-LhSorTGA2, was generated by cloning the LhSorTGA2 ORF into the pGADT7 vector after amplification with primers (LhSorTGA2-GADT7-F, LhSorTGA2-GADT7-R; Table 1). Constructed vectors were co-transformed into AH109 competent yeast cells in pairs, and transformants were plated on DDO and QDO mediums to test protein interactions. Transformants were also streaked on QDO medium supplemented with $40 \mu \mathrm{g} / \mathrm{ml}$ 5-bromo-4-chloro3 -indoxyl- $\alpha$-D-galactopyranoside (X- $\alpha-G a l)$ to further confirm interaction of different co-transformants.

\section{Gene expression analysis by $\mathrm{qPCR}$}

For tissue-specific expression of LhSorTGA2, root, stem, leaf, petal, and scale were sampled from 2-month-old fully bloomed plants and subjected to total RNA isolation as described above. For LhSorTGA2 expression in respond to hormone or pathogen treatment, lily seedlings of 1-month-old were subjected to $20 \mathrm{mM}$ methyl jasmonate (MeJA), $10 \mathrm{mM}$ sodium salicylate (SA), $5 \mathrm{mM}$ ethephon (ETH), $2 \mu \mathrm{M}$ abscisic acid (ABA) or B. elliptica $\left(8 \times 10^{5}\right.$ conidia $\left./ \mathrm{ml}\right)$ inoculation treatments. Preparation of phytohormone solutions, the B. elliptica inoculum, and the corresponding mock treatments were performed according our previous report (Wang et al. 2017a). Leaves were sampled at $0,2,4,8,12$, and 24 h post treatments, and froze immediately in liquid nitrogen and kept in a refrigerator at $-80^{\circ} \mathrm{C}$ ) until use.

After RNA extraction, 500 ng of total RNA was reversely transcribed to cDNA with the HiScript II Q RT SuperMix for qPCR kit (Vazyme Biotech, Nanjing, China). LhSorTGA2 specific primers (LhSorTGA2 qPCR-F, LhSorTGA2 qPCR-R; Table 1) were used for qPCR amplification, and the housekeeping gene polyubiquitin 4 was used as an internal reference (Wang et al. 2017c). All PCR reactions were conducted in a MX3000P qPCR thermocycler system (Stratagene Corp., USA). The following protocol 
was used for amplification: pre-denaturation for $10 \mathrm{~min}$ at $95^{\circ} \mathrm{C}$, followed by 40 cycles of $94^{\circ} \mathrm{C}$ for $15 \mathrm{~s}, 55^{\circ} \mathrm{C}$ for $15 \mathrm{~s}$, and $72{ }^{\circ} \mathrm{C}$ for $30 \mathrm{~s}$. The relative expression software tool (REST) was used for gene expression data analysis, and we applied $\mathrm{P}(\mathrm{H} 1)$ testing for statistical analysis. The Origin Pro 8.1 software was used for data graphing.

\section{Results}

Molecular cloning and sequence analysis of LhSorTGA2

The partial coding sequence of $L h S o r T G A 2$ was retrieved from sequences annotated in our previous RNA-seq data. Gene specific primers were designed for $5^{\prime}$ RACE and $3^{\prime}$ RACE PCR to obtain the LhSorTGA2 full-length sequence. DNA fragments amplified during RACE cloning were shown in Additional file 1: Figure S1. The LhSorTGA2 cloned was 2010 bp in length, comprising a $5^{\prime}$ untranslated region (UTR) of $337 \mathrm{bp}$, a predicted open reading frame (ORF) of $1278 \mathrm{bp}$, and a 3' UTR of $395 \mathrm{bp}$. The ORF encoded a protein of 425 amino acids with a theoretical molecular mass of $47.34 \mathrm{kDa}$ and a predicted isoelectric point (pI) of 6.74. The LhSorTGA2 full-length cDNA sequence has been submitted to GenBank and deposited under the Accession Number MF685037.

Alignment of LhSorTGA2 protein sequence to TGAs in Arabidopsis thaliana revealed that LhSorTGA2 was highly similar to members of the TGA family protein (Fig. 1). LhSorTGA2 contained the feature structures of bZIP transcription factors: a DNA binding domain (DBD) and a leucine zipper that enables protein dimerization. Within the DBD, glutamine, alanine, and serine residues, which were highly conserved for all TGA homologues were identified. Phosphorylation of the serine residue within the DBD led to DNA binding impediment, suggesting that phosphorylation might involve in the fine tune of transcription control of LhSorTGA2-regulated downstream genes (Kirchler et al. 2010). Leucine zippers of TGA proteins were formed by four heptads, within which three conserved leucine residues and one glycine residue were identified (Fig. 1). Two glutamine rich acid domains (QI and QII), which functioned in transcription activation, were also found in LhSorTGA2. The signature sequence for group D bZIP transcription factors, $\mathrm{Yx}_{2} \mathrm{RL}[\mathrm{RQ}] \mathrm{ALSS}[\mathrm{LS}] \mathrm{W}$, was identified at the C-terminus of all TGA homologues. A phylogenetic tree was built to analyze the evolutionary relationship of LhSorTGA2 with other TGA family proteins in plants. As shown in Fig. 2, TGA family proteins were subdivided into three clades, and LhSorTGA2 was most closely related to GhTGA2 in subclade I.

Subcellular localization of LhSorTGA2 and LhSorNPR1, and protein-protein interaction validated by BIFC

LhSorTGA2 and LhSorNPR1 (GenBank Accession: KY073343) were fused to the $\mathrm{N}$-terminus of GFP respectively, and the fusion proteins were transiently expressed in $N$. benthamiana leaf epidermal cells under the control of CaMV $35 \mathrm{~S}$ promoter to examine their subcellular localization. As shown in Fig. 3a, fluorescence of GFP-tagged LhSorTGA2 was predominantly detected in the nucleus. Different from LhSorTGA2, fluorescence of the LhSorNPR1-GFP infusion protein was observed both in the nucleus and at the cytomembrane. Strong green fluorescence was detected throughout the entire cell for the pCAMBIA1302-GFP control as expected. Our observation of subcellular localization of LhSorTGA2 and LhSorNPR1 was in agreement with their corresponding orthologues in Gladiolus hybridus from previous report (Zhong et al. 2015).

Protein-protein interaction between NPR1 and TGA2 was first identified in Arabidopsis. To test whether the interaction between LhSorNPR1 and LhSorTGA2 exists or not, we performed BIFC experiment by co-expressing LhSorNPR1-YFP ${ }^{\mathrm{N}}$ and LhSorTGA2-YFP ${ }^{\mathrm{C}}$ transiently in $N$. benthamiana leaf epidermal cells. As shown in Fig. 3b, strong fluorescence was detected in the LhSorNPR1$\mathrm{YFP}^{\mathrm{N}}$ and LhSorTGA2-YFP ${ }^{\mathrm{C}}$ co-infiltration treatment, indicating reconstitution of YFP fluorophore by the interaction between LhSorNPR1 and LhSorTGA2. In contrast, co-infiltration of LhSorNPR1-YFP ${ }^{\mathrm{N}}$ and $\mathrm{YFP}^{\mathrm{C}}$ or YFP $^{\mathrm{N}}$ and LhSorTGA2-YFP ${ }^{\mathrm{C}}$, did not produce any detectable fluorescent signal. These findings indicate that LhSorNPR1 interacts with LhSorTGA2.

Confirmation of LhSorNPR1-LhSorTGA2 interaction by Y2H To further confirm the interaction between LhSorTGA2 and LhSorNPR1, the prey plasmid pGADT7LhSorTGA2 and the bait plasmid pGBKT7-LhSorNPR1 were co-transformed into AH109 competent yeast cells. Transformants were selected on DDO and QDO mediums, and protein-protein interaction between LhSorTGA2 and LhSorNPR1 was identified (Fig. 4a). In contrast, no positive interaction was detected in a series of co-transformants used as negative controls. All co-transformants were streaked on QDO medium supplemented with $\mathrm{X}-\alpha-\mathrm{Gal}$ chromogenic substrate, and blue colonies were only observed for the pGADT7LhSorTGA2 and pGBKT7-LhSorNPR1 co-transformant and the positive control (pGADT7-T and pGBKT7-53 co-transformant) (Fig. 4b ,c).

Expression analysis of LhSorTGA2 in different tissues and in response to pathogen infection and various hormone treatments

Expression of LhSorTGA2 was detected in all the five tissues explored, but the transcript abundance varied from tissue to tissue (Fig. 5). The transcript levels of LhSorTGA2 observed in leaf, petal, root, and scale, were 


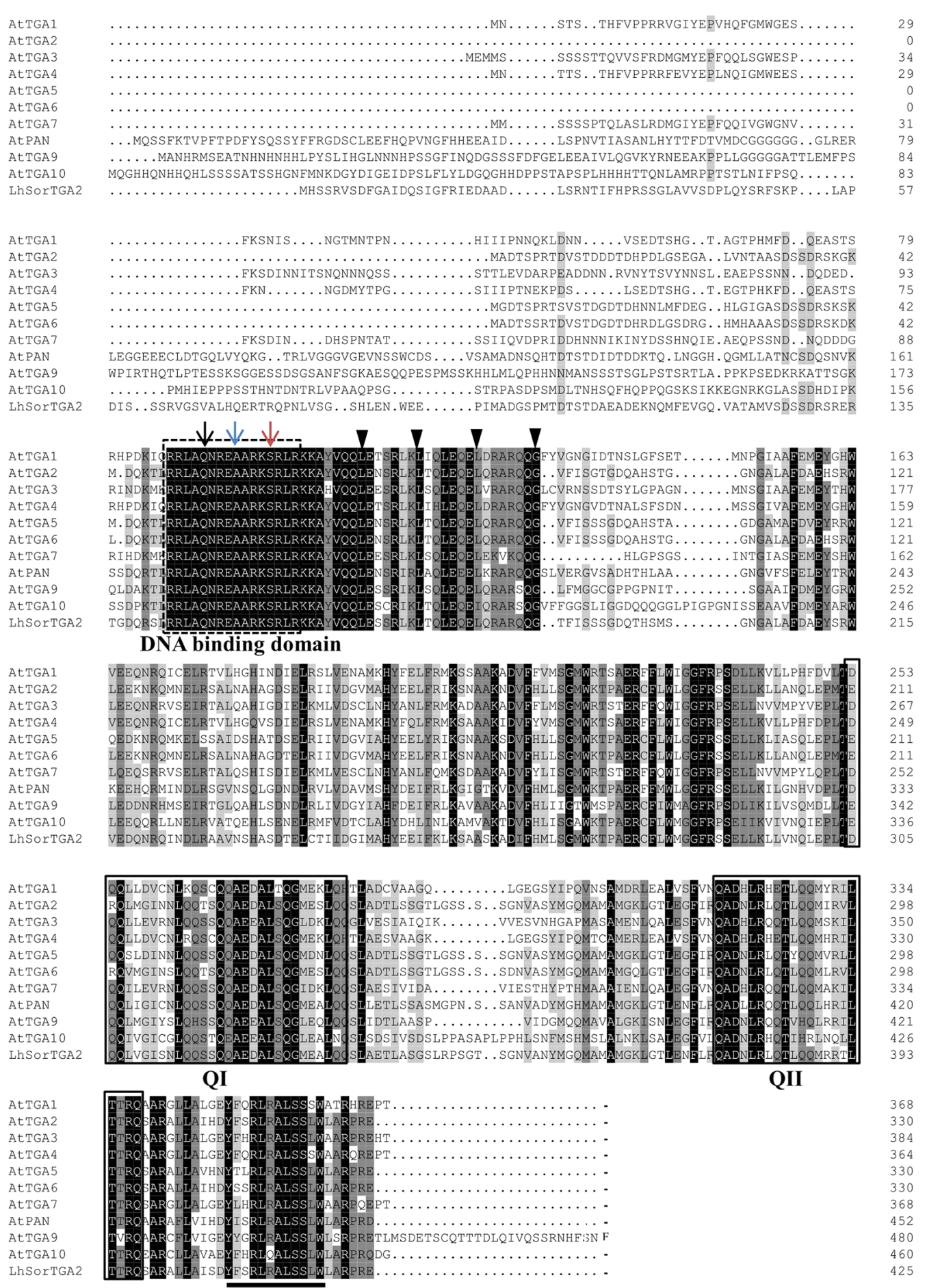

Fig. 1 Sequence alignment of LhSorTGA2 with proteins of the TGA family in Arabidopsis. Sequences were aligned using Clustal W. Identical residues are shaded in black, highly similar residues are shaded in dark gray, and similar residues are shaded in gray. The DNA binding domain (DBD) is indicated by a dashed line box. The conserved glutamine residues (black arrow), alanine residues (blue arrow) and serine residues (red arrow), are indicated by arrows. Three leucine residues and one glycine residue for the four heptad repeats in leucine zipper are highlighted by triangles. Two glutamine rich acid domains (QI and QII) that mediate transcription activation are highlighted by solid line boxes. The signature sequence for group $\mathrm{D}$ bZIP transcription factors, $\mathrm{Y}_{2} \mathrm{RL}[\mathrm{RQ}] \mathrm{ALSS}[\mathrm{LS}] \mathrm{W}$, is underlined. The accession numbers of all the TGA proteins used for sequence alignment are listed in Additional file 2: Table S1 


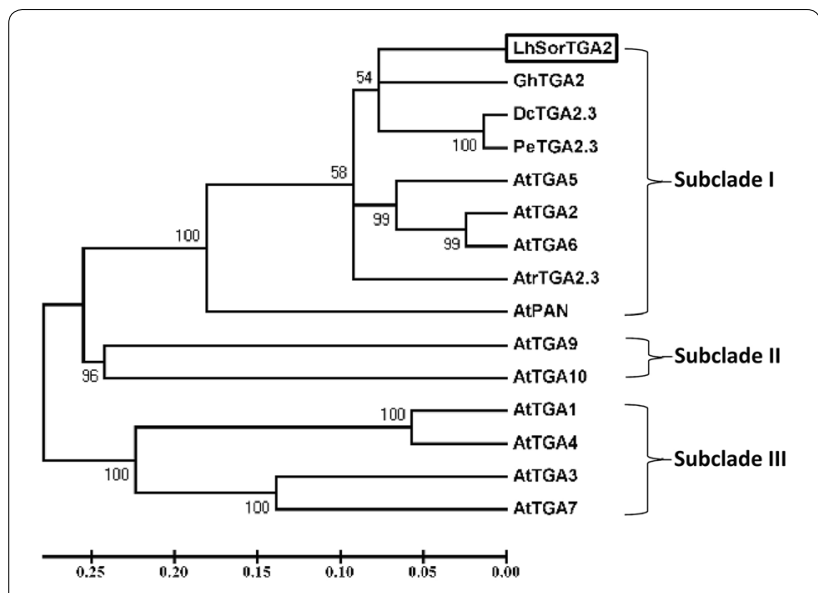

Fig. 2 Phylogenetic relationship of LhSorTGA2 with TGA family proteins from other plants. The phylogram was constructed using the neighbor-joining method in Mega 5, and tested with 1000 bootstrap iterations. The Accession Numbers of all the TGA proteins used for phylogram construction are listed in Additional file 2: Table S1 all lower than that in stem, making stem the most abundant tissue expressing LhSorTGA2.

To investigate LhSorTGA2 expression in respond to pathogen infection or hormone treatments, transcript levels of $L h S o r T G A 2$ were quantified after subjected to different treatments (MeJA, SA, ETH, ABA, and $B$. elliptica infection). As shown in Fig. 6a, when subjected to MeJA treatment, LhSorTGA2 transcription was significantly induced, reaching its peak level $4 \mathrm{~h}$ after treatment. Foliar spray of SA also stimulated the accumulation of LhSorTGA2, resulting in a 4.15 times increase of LhSorTGA2 transcript level $24 \mathrm{~h}$ after treatment (Fig. 6b). Following the ETH treatment, LhSorTGA2 was up-regulated 4.02 times the control level (Fig. 6c). For the ABA treatment, LhSorTGA2 expression was rapidly induced $2 \mathrm{~h}$ post treatment, reaching a level 2.17 times that of in the control. When exposed to pathogenic fungus B. elliptica, the expression of LhSorTGA2 was also up-regulated, reaching its highest level $12 \mathrm{~h}$ after treatment (by a factor of 2.77 compared to the control). Upregulation of LhSorTGA2 in respond to fungal infection and hormone treatments suggested that LhSorTGA2 might involve in diverse defense pathways regulated by multiple phytohormones.

\section{Discussion}

Transcriptional reprogramming during SAR is predominantly mediated by NPR1. The interactions between NPR1 and TGA family proteins modulate expression of $P R$ genes during SAR. bZIP TFs in Arabidopsis are subdivided into 10 groups (A-I and S), among which group

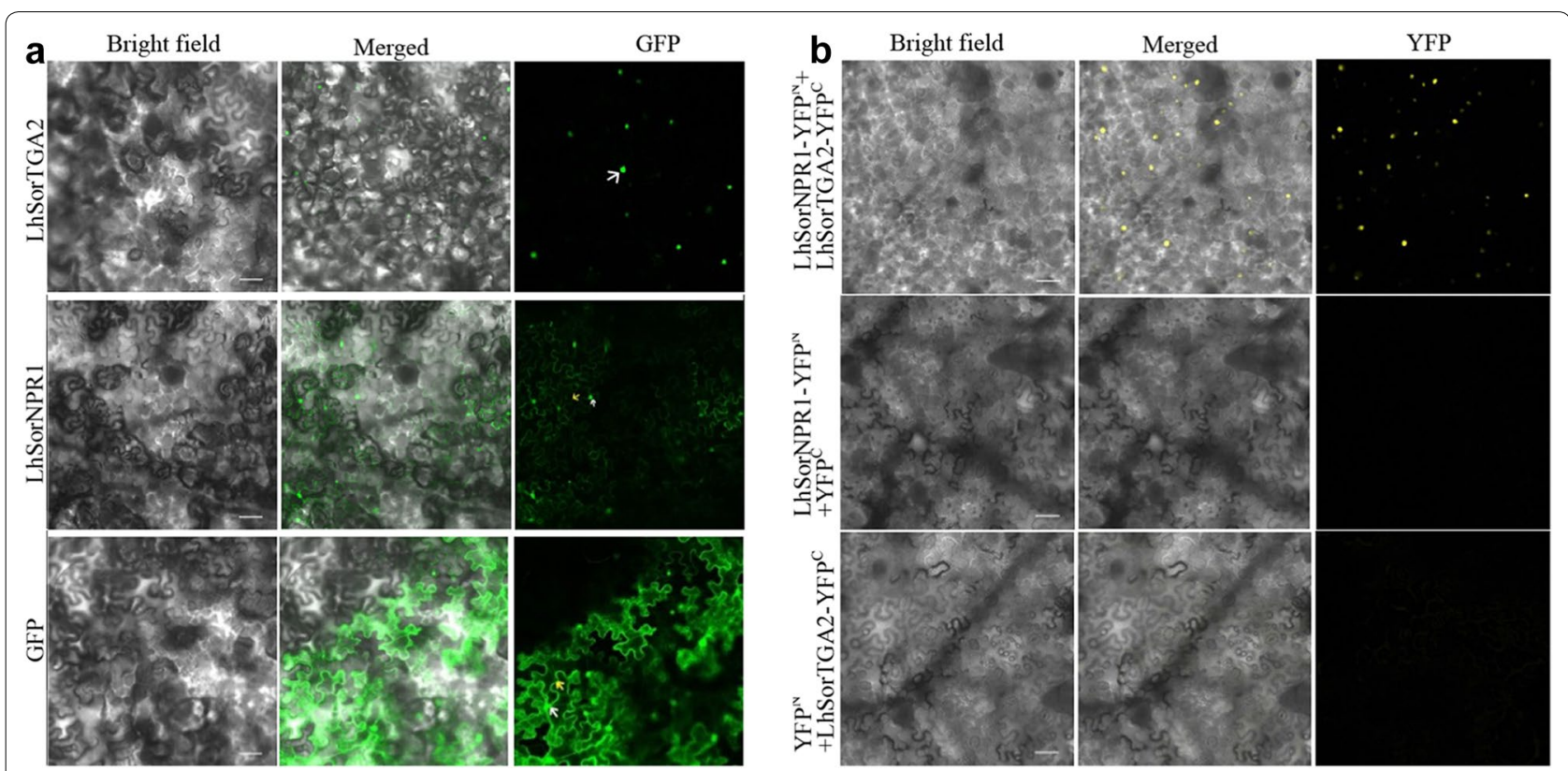

Fig. 3 Subcellular localization of LhSorTGA2 and LhSorNPR1, and their interaction validated by BIFC in planta. a Localization of LhSorTGA2 and LhSorNPR1 in N. benthamiana leaf epidermal cells. b BIFC analysis to validate the protein interaction between LhSorTGA2 and LhSorNPR1. LhSorNPR1 was fused to the N-terminal, while LhSorTGA2 was fused to the C-terminal half of YFP protein. Yellow fluorescence indicated protein interaction between LhSorTGA2 and LhSorNPR1 in the nuclei. Fluorescence was observed by confocal microscopy, and bright field and merged images were also shown for each observation. White arrows indicate nuclei of epidermal cells, and yellow arrows indicate the cytomembrane. $\operatorname{Bar}=100 \mu \mathrm{m}$ 


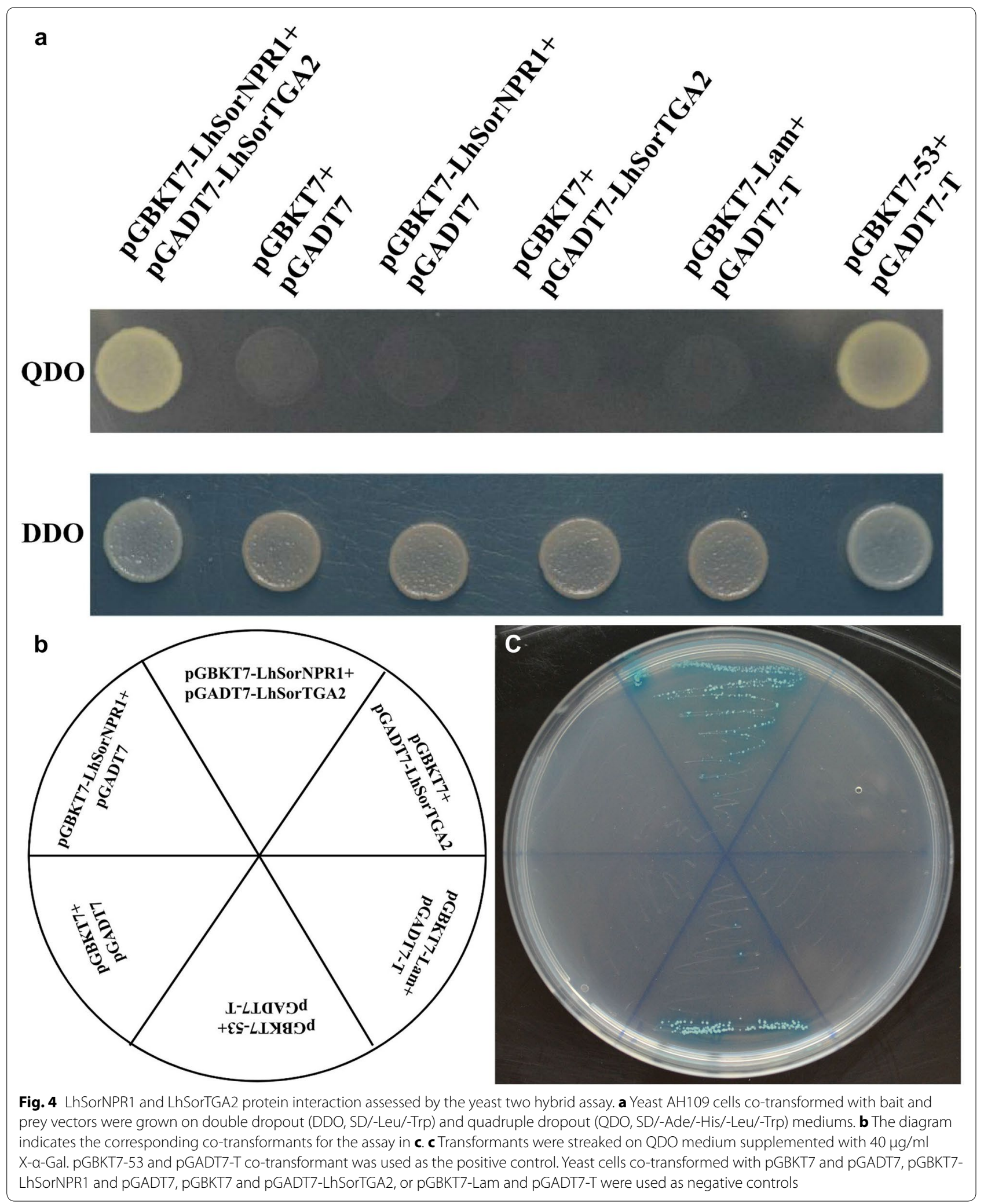




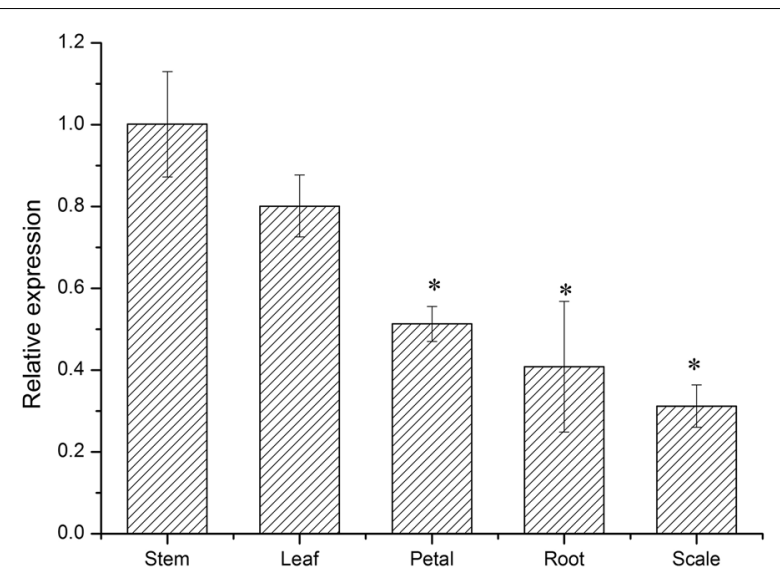

Fig. 5 Tissue-specific expression of LhSorTGA2 in oriental hybrid lily CV. Sorbonne. The expression of LhSorTGA2 in stem was used as calibrator (designated as 1.0) to determine the relative expression of the target gene in different tissues. Relative expression was calculated using REST 2009 software, and the values were presented as mean \pm standard deviation (SD) for three replicates. Bars labeled with an asterisk indicate significant differences from the calibrator at $\mathrm{P}<0.05$ (the REST statistical randomization test)

D proteins are crucial participants in response to pathogen infection (Jakoby et al. 2002). Previous survey of the Arabidopsis genome identified 10 members of the group D bZIP TFs (TGA family proteins), of which seven have been confirmed to exist interaction with NPR1 (Kesarwani et al. 2007).

Lilies are one of the most important floriculture crops in cut flower market and are valued for their elegant flowers emitting fragrance. However, a lack of genome information hinders the genetic improvement targeting genes of interest, for instance, genes related to ornamental traits or disease resistance. Species in the genus Lilium have huge genomes (around $36 \mathrm{~GB}$ ), which makes sequencing and assembly of their genomes a technical challenge (Du et al. 2017). In light of the constraint in availability of the genomic data, RNA-seq seems to be the most practical approach to profile the transcriptional change of genes of interest in lily. In the present study, the partial coding sequence of LhSorTGA2 was retrieved from our previous transcriptome sequencing data, and the LhSorTGA2 full-length cDNA was obtained by RACE. Subsequent in silicon analysis confirmed the homology of LhSorTGA2 to TGA-like proteins in other plants, such as AtTGA2 (72.23\% similarity) in Arabidopsis and GhTGA2 (85.55\% similarity) in Gladiolus hybrid cultivar. Sequence alignment of LhSorTGA2 with TGA proteins in Arabidopsis revealed the structural basis for TGA2-mediated transcription control. The leucine zipper domain determines the dimerization specificity of bZIP TFs (Vinson et al. 2006), forming homo- or heterodimeric coiled-coil structures (zipper). The contiguous basic region, which contains a predicted nuclear localization signal (amino acids 143-159 in LhSorTGA2), contacts the DNA. Whereas the glutamine rich acid domains (QI and QII) have been proven to transactivate PR1 expression in a NPR1-dependent way during SAR (Fan and Dong 2002).

A phylogenetic tree was built to characterize the evolutionary relationship of TGA-like proteins originated from different species. LhSorTGA2 was grouped in subcladed I, together with AtTGA2, AtTGA5 and AtTGA6. These three closely related TGAs have been proposed as transcriptional repressors of $P R 1$, which are required for the basal repression of $P R 1$. However, in the state of SAR, their biological functions are both redundant and essential, since only the tga 2 tga 5 tga 6 triple mutant is blocked from induction of PR1 during SAR (Zhang et al. 2003).

To further investigate the function of LhSorTGA2 and LhSorNPR1, subcellular localization of GFP-tagged proteins was examined using confocal microscopy. As mentioned above, a NLS was predicted in the middle of LhSorTGA2, which probably making it a nucleuslocalized protein. Consistent with the prediction, GFPtagged LhSorTGA2 was predominately detected in the nucleus. A NLS was also predicted in LhSorNPR1 at the $\mathrm{C}$ terminus, but presence of GFP-tagged LhSorNPR1 was detected both in the nucleus and at the cytomembrane. Consecutive expression of GFP-tagged AtNPR1 or TcNPR1 in Arabidopsis has proven that NPR1 localized in the cytoplasma if the seedlings were not treated with SA (Mou et al. 2003; Shi et al. 2010). However, under SA treatment (in the state of SAR), both AtNPR1-GFP and TcNPR1-GFP fusion proteins were translocated into the nucleus. Agroinfiltration causes immune response. Hence, SAR might be triggered during subcellular localization of GFP-labeled proteins using tabacco transient expression system. Our observation of LhSorNPR1GFP in the nucleus was consistent with previous reports (GhNPR1 and VvNPR1), which might be caused by the translocation of LhSorNPR1-GFP into the nucleus in the state of SAR during agroinfiltration (Le Henanff et al. 2009; Zhong et al. 2015).

In Arabidopsis, interactions between NPR1 and TGA2 clade proteins modulated the expression of PR1. To validate the possible interaction between LhSorTGA2 and LhSorNPR1, vectors were constructed to test interaction in two different systems. As shown in Figs. 3 and 4, both $\mathrm{BIFC}$ and $\mathrm{Y} 2 \mathrm{H}$ tests confirmed the interaction between LhSorTGA2 and LhSorNPR1. However, whether transcription of $P R$ genes, especially $P R 1$, is regulated by LhSorTGA2-LhSorNPR1 interaction in lily still needs to be confirmed.

Expression of LhSorTGA2 was detected in all the five tissues (leaf, stem, root, petal and scale) explored, but 

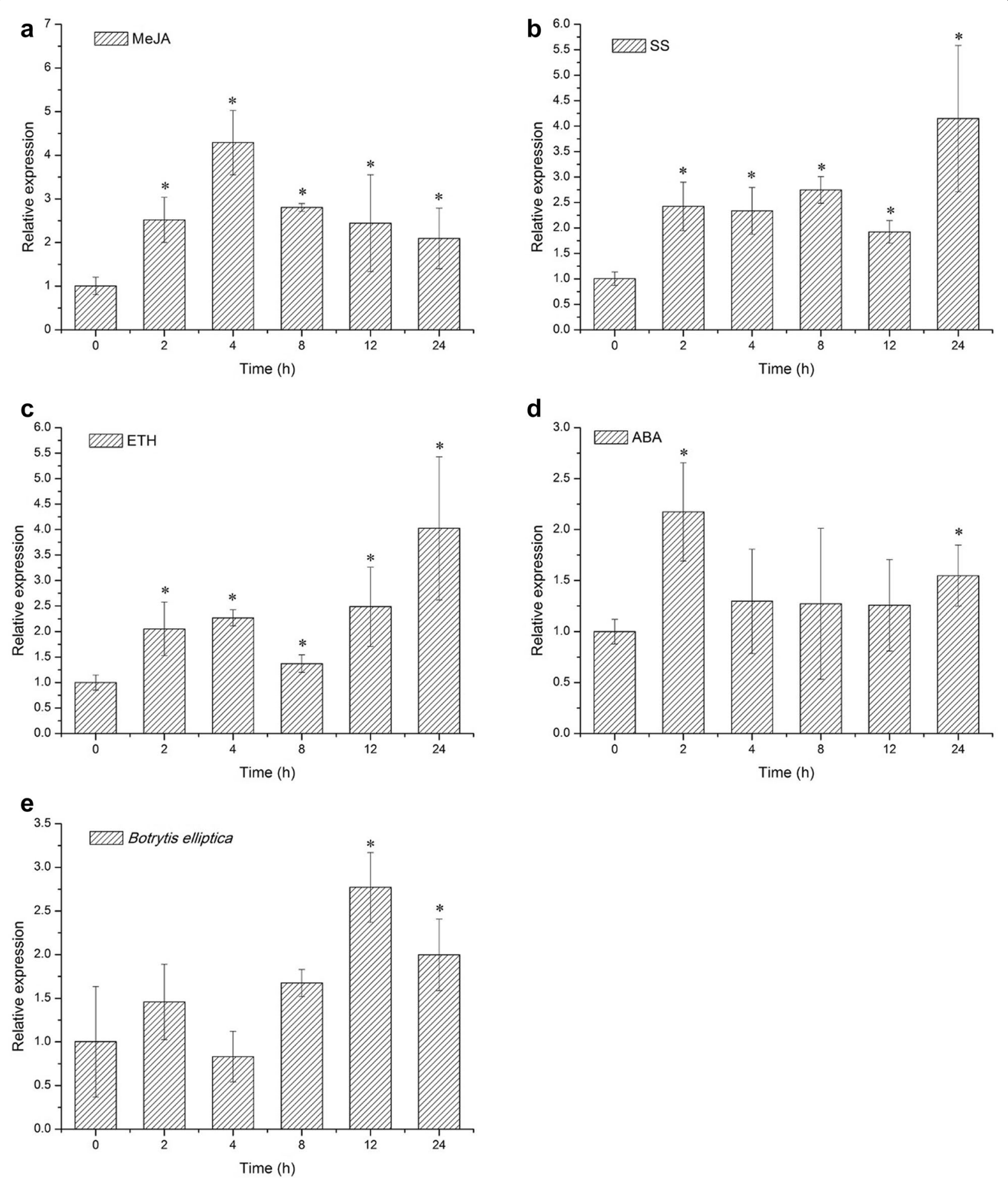

Fig. 6 LhSorTGA2 expression in respond to a methyl jasmonate (MeJA), b sodium salicylate (SS), c ethephon (ETH), d abscisic acid (ABA) and e Botrytis elliptica inoculation treatments. Foliar spray of MeJA, SS, ETH, ABA, or Spores of B. elliptica $\left(8 \times 10^{5} \mathrm{conidia} / \mathrm{ml}\right)$, was applied to 1-month-old lily seedling. Leaves were sampled $0,2,4,8,12,24 \mathrm{~h}$ post treatments to explore LhSorTGA2 expression. Expression at $0 \mathrm{~h}$ was used as the calibrator (designated as 1.0) to determine the relative expression of the target gene at different time points. Values are shown as mean \pm standard deviations (SD) for three replicates. Bars labeled with an asterisk indicate significant differences from the control $(0 \mathrm{~h}$ ) at $\mathrm{P}<0.05$ (the REST statistical randomization test) 
the transcription levels varied substantially (Fig. 5). TGA2 participated in SA-regulated SAR induction, and the expression of TGA2 itself has been shown to be responsive to SA in Arabidopsis (Fan and Dong 2002). In our case, LhSorTGA2 expression was responsive to SA, MeJA, ETH, ABA, and B. elliptica inoculation treatments, implying diverse roles it might play in defense pathways regulated by different hormones. Phytohormones are tightly linked to physiological processes in plant (Weyers and Paterson 2001). Induction of LhSorTGA2 in respond to multiple hormones suggested that LhSorTGA2 might relate to physiological processes contributing to plant defense. Although there is still no direct evidence linking expression of TGA2-like genes to physiological changes under pathologic conditions, AtTGA10 and AtTGA9, two other members of the TGA family in Arabidopsis, has been shown to be involved in reactive oxygen species (ROS)-mediated responses to bacterial PAMP flg22 (Noshi et al. 2016). Accumulation of ROS is associated with hypersensitive response (HR) that restricts the spread of pathogens (Zurbriggen et al. 2010). However, whether TGA family proteins in lily modulate disease resistance through ROS accumulation is unknown. Among these TGA proteins, which one is the key regulator involved in ROS-mediated $\mathrm{HR}$ response to pathogen? Answers to these questions are mysteries awaited to be uncovered in future research.

\section{Conclusions}

LhSorTGA2, a novel TGA-like protein, was identified as an interacting partner of LhSorNPR1 in lily. Sequence alignment revealed that LhSorTGA2 was a member of the group D bZIP transcription factors, which featured with a conserved bZIP domain and two glutamine rich acid domains. LhSorTGA2 was differentially expressed in various tissues, and its expression was responsive to SA, MeJA, ETH, ABA, and B. elliptica inoculation treatments. However, whether transcription of $P R$ genes is modulated by the interaction between LhSorTGA2 and LhSorNPR1 still needs to be tested.

\section{Additional files}

Additional file 1: Figure S1. Cloning of the LhSOrTGA2 gene by RACE. a partial coding sequence of LhSOrTGA2; b 5' LhSOrTGA2 RACE PCR products; c 3' LhSorTGA2 RACE PCR products; d The LhSorTGA2 open reading frame amplified. M: DNA marker.

Additional file 2: Table S1. TGA-like protein sequences retrieved from GenBank used for phylogram construction and sequence alignment.

\section{Abbreviations}

SAR: systemic acquird resistance; NPR1: non-expressor of pathogenesis-related genes 1; bZIP: basic leucine-zipper; RACE: rapid amplification of CDNA ends; BIFC: bimolecular fluorescence complementation; $\mathrm{Y} 2 \mathrm{H}$ : yeast two-hybrid;
QDO: SD/-Ade/-His/-Leu/-Trp; DDO: SD/-Leu/-Trp; ABA: abscisic acid; SA: salicylic acid; SS: sodium salicylate; MeJA: methyl jasmonate; ETH: ethephon.

\section{Authors' contributions}

$Z X$ and YW conceived and designed the experiments; LW and ZG performed the experiments; YZ, LW and WL analyzed the data; LW, GY and RW wrote the manuscript. All authors read and approved the final manuscript.

\section{Author details}

${ }^{1}$ Gaolan Station of the Agricultural and Ecological Experiment, Northwest Institute of Eco-Environment and Resources, Chinese Academy of Sciences, Lanzhou 730000, China. ${ }^{2}$ University of Chinese Academy of Sciences, Beijing 100049, China. ${ }^{3}$ The Forest Tree Seedling Station of the Alxa League, Alxa League 750300 , China.

\section{Competing interests}

The authors declare that they have no competing interests.

\section{Availability of data and materials}

The data and material obtained in this paper are available.

Consent for publication

Not applicable.

Ethics approval and consent to participate

Not applicable.

\section{Funding}

This work was supported by the Ningxia Agricultural Comprehensive Development Office (NTKJ2015-05-01), the National Natural Science Foundation of China (No. 31101190 and No. 31201651), the CAS Key Technology Talent Program (No. 2016-65), the "West Light" project of the Chinese Academy of Sciences in 2014, the China Postdoctoral Science Foundation project (No. 2015M580894), and the Hundred Talents Programs of Chinese Academy of Sciences (No. Y629721002 and No. 27Y127L41002).

\section{Publisher's Note}

Springer Nature remains neutral with regard to jurisdictional claims in published maps and institutional affiliations.

Received: 1 September 2017 Accepted: 6 November 2017

Published online: 10 November 2017

\section{References}

Cao H, Glazebrook J, Clarke JD, Volko S, Dong XN (1997) The Arabidopsis NPR1 gene that controls systemic acquired resistance encodes a novel protein containing ankyrin repeats. Cell 88:57-63

Du YP, Bi Y, Zhang MF, Yang FP, Jia GX, Zhang XH (2017) Genome size diversity in Lilium (Liliaceae) is correlated with karyotype and environmental traits. Front Plant Sci 8:1303

Fan WH, Dong XN (2002) In vivo interaction between NPR1 and transcription factor TGA2 leads to salicylic acid-mediated gene activation in Arabidopsis. Plant Cell 14:1377-1389

Fu ZQ, Dong X (2013) Systemic acquired resistance: turning local infection into global defense. Annu Rev Plant Biol 64:839-863

Gaffney T, Friedrich L, Vernooij B, Negrotto D, Nye G, Uknes S, Ward E, Kessmann H, Ryals J (1993) Requirement of salicylic-acid for the induction of systemic acquired-resistance. Science 261:754-756

Gao QM, Zhu S, Kachroo P, Kachroo A (2015) Signal regulators of systemic acquired resistance. Front Plant Sci 6:228

Gatz C (2013) From pioneers to team players: TGA transcription factors provide a molecular link between different stress pathways. Mol Plant Microbe Interact 26:151-159

Huang CJ, Liu YH, Yang KH, Chen CY (2012) Physiological response of Bacillus cereus C1L-induced systemic resistance in lily against Botrytis leaf blight. Eur J Plant Pathol 134:1-12 
Jakoby M, Weisshaar B, Droge-Laser W, Vicente-Carbajosa J, Tiedemann J, Kroj T, Parcy F (2002) bZIP transcription factors in Arabidopsis. Trends Plant Sci 7:106-111

Kerppola TK (2013) Bimolecular fluorescence complementation (BiFC) analysis of protein interactions in live cells. Cold Spring Harbor Protoc 2013:727-731

Kesarwani M, Yoo J, Dong X (2007) Genetic interactions of TGA transcription factors in the regulation of pathogenesis-related genes and disease resistance in Arabidopsis. Plant Physiol 144:336-346

Kirchler T, Briesemeister S, Singer M, Schutze K, Keinath M, Kohlbacher O, Vicente-Carbajosa J, Teige M, Harter K, Chaban C (2010) The role of phosphorylatable serine residues in the DNA-binding domain of Arabidopsis bZIP transcription factors. Eur J Cell Biol 89:175-183

Le Henanff G, Heitz T, Mestre P, Mutterer J, Walter B, Chong J (2009) Characterization of Vitis vinifera NPR1 homologs involved in the regulation of pathogenesis-related gene expression. BMC Plant Biol 9:54

Lecomte C, Alabouvette C, Edel-Hermann V, Robert F, Steinberg C (2016) Biological control of ornamental plant diseases caused by Fusarium oxysporum: a review. Biol Control 101:17-30

Mou Z, Fan WH, Dong XN (2003) Inducers of plant systemic acquired resistance regulate NPR1 function through redox changes. Cell 113:935-944

Noshi M, Mori D, Tanabe N, Maruta T, Shigeoka S (2016) Arabidopsis clade IV TGA transcription factors, TGA10 and TGA9, are involved in ROS-mediated responses to bacterial PAMP flg22. Plant Sci 252:12-21

Pieterse CM, Van Loon L (2004) NPR1: the spider in the web of induced resistance signaling pathways. Curr Opin Plant Biol 7:456-464

Shi Z, Maximova SN, Liu Y, Verica J, Guiltinan MJ (2010) Functional analysis of the Theobroma cacao NPR1 gene in Arabidopsis. BMC Plant Biol 10:248

Tamura K, Peterson D, Peterson N, Stecher G, Nei M, Kumar S (2011) MEGA5: molecular evolutionary genetics analysis using maximum likelihood, evolutionary distance, and maximum parsimony methods. Mol Biol Evol 28:2731-2739

Vinson C, Acharya A, Taparowsky EJ (2006) Deciphering B-ZIP transcription factor interactions in vitro and in vivo. BBA Gene Struct Expr 1759:4-12
Wang L, Li LJ, Zhang YB, Guo ZH, Wang YJ, Yang G, Wang L, Wang RY, Xie ZK (2017a) Characterization of LhSorPR5, a Thaumatin-like protein from the oriental hybrid lily Sorbonne. Int J Agric Biol 19:865-872

Wang L, Guo ZH, Zhang YB, Wang YJ, Yang G, Yang L, Wang L, Wang RY, Xie ZK (2017b) Overexpression of LhSorNPR1, a NPR1-like gene from the oriental hybrid lily'Sorbonne', conferred enhanced resistance to Pseudomonas syringae pv. tomato DC3000 in Arabidopsis. Physiol Mol Biol. https://doi. org/10.1007/s12298-017-0466-3

Wang L, Guo ZH, Zhang YB, Wang YJ, Yang G, Yang L, Wang RY, Xie ZK (2017C) Characterization of LhSorP5CS, a gene catalyzing proline synthesis in oriental hybrid lily Sorbonne: molecular modelling and expression analysis. Bot Stud 58:10

Wang L, Guo ZH, Zhang YB, Wang YJ, Yang G, Yang L, Wang RY, Xie ZK (2017d) Isolation and characterization of two distinct class II PR4 genes from the oriental lily hybrid Sorbonne. Russ J Plant Physiol. 64:707-717

Weyers JDB, Paterson NW (2001) Plant hormones and the control of physiological processes. New Phytol 152:375-407

Zhang YL, Fan WH, Kinkema M, Li X, Dong XN (1999) Interaction of NPR1 with basic leucine zipper protein transcription factors that bind sequences required for salicylic acid induction of the PR-1 gene. Proc Natl Acad Sci USA 96:6523-6528

Zhang YL, Tessaro MJ, Lassner M, Li X (2003) Knockout analysis of Arabidopsis transcription factors TGA2, TGA5, and TGA6 reveals their redundant and essential roles in systemic acquired resistance. Plant Cell 15:2647-2653

Zhang YB, Wang YJ, Xie ZK, Wang RY, Guo ZH, van der Werf W, Wang L (2016) Purification and immuno-gold labeling of lily mottle virus from lily leaves. J Virol Methods 232:33-38

Zhong XH, Xi L, Lian QL, Luo X, Wu Z, Seng SS, Yuan X, Yi MF (2015) The NPR1 homolog GhNPR1 plays an important role in the defense response of Gladiolus hybridus. Plant Cell Rep 34:1063-1074

Zurbriggen MD, Carrillo N, Hajirezaei MR (2010) ROS signaling in the hypersensitive response: when, where and what for? Plant Signal Behav 5:393-396

\section{Submit your manuscript to a SpringerOpen ${ }^{\circ}$ journal and benefit from:}

- Convenient online submission

- Rigorous peer review

- Open access: articles freely available online

- High visibility within the field

Retaining the copyright to your article

Submit your next manuscript at $\boldsymbol{\nabla}$ springeropen.com 